\title{
TENSION FIELD AND INDEX FORM OF ENERGY-TYPE FUNCTIONALS
}

\author{
STEFAN BECHTLUFT-SACHS \\ Universität Regensburg, Fakultät für Mathematik, D-93040 Regensburg, Germany \\ e-mail: stefan_bechtluft-sachs@mathematik-uni-regensburg.de
}

(Received 20 September, 2001; accepted 28 March, 2002)

\begin{abstract}
We derive variational formulae for natural first order energy functionals and obtain criteria for the stability of isometric immersions. This generalizes known results for the classical energy, the $p$-energy and the exponential energy.

2000 Mathematics Subject Classification. AMS Subject Classification: 58E20 (53C43).
\end{abstract}

1. Introduction. By an energy-type functional defined on smooth maps $f:\left(M^{n}, g\right) \rightarrow\left(V^{k}, h\right)$ of compact Riemannian manifolds we mean a functional obtained by integration of a first order differential operator $\phi(d f)$ where $d f \in \Gamma\left(T^{*} M \otimes\right.$ $\left.f^{*} T V\right)$ denotes the differential of $f$ and $\phi: M(\mathbb{R}, n \times k) \rightarrow \mathbb{R}_{0}^{+}$is invariant under the action of $O(n) \times O(k)$. Especially $\phi$ yields a parallel function $T^{*} M \otimes f^{*} T V \rightarrow \mathbb{R}_{0}^{+}$. We can rewrite $\phi(d f)=\Phi\left(d f^{*} d f\right)$ for some function $\Phi: M(\mathbb{R}, n \times n)^{+} \rightarrow \mathbb{R}$ on nonnegative symmetric matrices which is invariant under conjugation by $O(n)$. The functionals in question take the form

$$
E_{\Phi}(f):=\int_{M} \Phi\left(d f^{*} d f\right) d \mathrm{vol}_{g}
$$

where we have used the Riemannian metrics to identify $T^{*} M=T M$ and $T^{*} V=T V$ to get the endomorphism $d f^{*} d f$ of $T M$.

Famous examples of this construction are the classical energy, $\Phi(A)=\operatorname{Tr} A$, the exponential energy, $\Phi(A)=\exp (\operatorname{Tr} A)$ as in [7], the $p$-energy, $\Phi(A)=(\operatorname{Tr} A)^{p}$ but also the volume, where $\Phi(A)=(\operatorname{det} A)^{1 / 2}$. Results similiar to ours in the case where $\Phi$ is a function of the Trace, $\Phi(A)=F(\operatorname{Tr} A)$, have been obtained in [1]. In particular the exponential energy was treated in [2] and the $p$-energy in [3]. There is a vast literature for the classical energy, see e.g. the survey papers $[\mathbf{5}, \mathbf{6}]$. For a discussion of stability results in this case we refer to [9] and the references there.

Here we will derive the first and second variational formulae for the $\Phi$-energy functional. The Bochner formula for vector fields then implies that isometries are $\Phi$-stable under certain conditions on the first and second derivative of $\Phi$. As in the classical case, (see [4], [9]) there is also a range of maps $\Phi$ such that the identity on the sphere $S^{n}$ is unstable for the $\Phi$-energy.

2. Variation formulae for the $\boldsymbol{\Phi}$-Energy. In order to derive variational formulae we will restrict ourselves to functionals which can be expressed with smooth $\Phi$, i.e. we work with $\Phi$ rather than $\phi$. This has the advantage that the domain $T M^{*} \otimes T M$ of $\Phi$ 
is independent of $f$. For polynomial (or even analytic) $\phi$ this is no loss of generality by the remark at the end of this section. In the sequel we will always assume $M$ compact or at least that the variations are compactly supported. Consider a 2-parameter variation of $f$, i.e. a map

$$
F: I \times J \times M \rightarrow V(s, t, m) \mapsto f_{s, t}(m)
$$

where $I, J$ are intervalls around 0 . Denote by $\nabla$ the Riemannian connections on the bundles $T M, F^{*} T V$ and $f^{*} T V$ and let $v:=d F\left(\frac{\partial}{\partial t}\right)=\frac{\partial}{\partial t} f_{s, t}(m), w:=d F\left(\frac{\partial}{\partial s}\right)=\frac{\partial}{\partial s} f_{s, t}(m)$ be the variation vector fields along $f=f_{0}=f_{0,0}, f_{t}=f_{0, t}$. We compute the variation at a point $p \in M$. Let $e_{1}, \ldots, e_{n}$ be a local orthonormal framing of $T M$ in a vicinity of $p$ with $\nabla_{e_{i}} e_{j}=0$ at $p$. Note that for the commutators we have $\left[e_{i}, \frac{\partial}{\partial s}\right]=0,\left[e_{i}, \frac{\partial}{\partial t}\right]=0$ and $\left[e_{i}, e_{j}\right](p)=0$. We also write $\bar{\partial}_{i, j} \Phi:=\partial_{i, j} \Phi+\partial_{j, i} \Phi$. In the subsequent calculations summation over the indices $i, j, k, l$ is tacitely assumed. For the first variation of the $\Phi$-energy density we obtain

$$
\begin{aligned}
\frac{d}{d t} \Phi\left(d f_{t}^{*} d f_{t}\right) & =d \Phi(\nabla d f \otimes d f+d f \otimes \nabla d f) \\
& =\bar{\partial}_{i, j} \Phi\left(d f^{*} d f\right)\left(\nabla_{\frac{\partial}{\partial t}} d F e_{i}\left|d F e_{j}\right\rangle\right. \\
& =\bar{\partial}_{i, j} \Phi\left(d f^{*} d f\right)\left\langle\nabla_{e_{i}} v \mid d f e_{j}\right\rangle \\
& =e_{i}\left(\bar{\partial}_{i, j} \Phi\left(d f^{*} d f\right)\left\langle v \mid d f e_{j}\right\rangle\right)-\left\langle v \mid \nabla_{e_{i}}\left(\bar{\partial}_{i, j} \Phi\left(d f^{*} d f\right) d f e_{j}\right)\right\rangle \\
& =\operatorname{div}\left(\left(\bar{\partial}_{i, j} \Phi\left(d f^{*} d f\right)\left\langle v \mid d f e_{j}\right\rangle\right) e_{i}\right)-\left\langle v \mid \tau_{\Phi}(f)\right\rangle .
\end{aligned}
$$

We thus get the

Proposition 2.1. Define the $\Phi$-tension of a smooth map $f: M \rightarrow V$ of compact Riemannian manifolds to be the vector field along $f$

$$
\begin{aligned}
\tau_{\Phi}(f) & :=\nabla_{e_{i}}\left(\bar{\partial}_{i, j} \Phi\left(d f^{*} d f\right) d f e_{j}\right) \\
& =\bar{\partial}_{k, l} \bar{\partial}_{i, j} \Phi\left(d f^{*} d f\right)\left(\nabla_{e_{i}} d f e_{k}\left|d f e_{l}\right\rangle d f e_{j}+\bar{\partial}_{i, j} \Phi\left(d f^{*} d f\right) \nabla_{e_{i}} d f e_{j}\right.
\end{aligned}
$$

Then $f$ is $\Phi$-harmonic, i.e. critical for the $\Phi$-energy, if and only if $\tau_{\Phi}(f)=0$.

For the second variation we get up to divergence

$$
\begin{aligned}
\frac{d^{2}}{d s d t} \Phi\left(d f_{s, t}^{*} d f_{s, t}\right)= & -\frac{d}{d s}\left\langle v \mid \tau_{\Phi}\left(f_{s}\right)\right\rangle \\
= & -\left\langle\nabla_{\frac{\partial}{\partial s}} v \mid \tau_{\Phi}(f)\right\rangle-\left\langle v \mid \nabla_{\frac{\partial}{\partial s}} \tau_{\Phi}\left(f_{s}\right)\right\rangle \\
= & -\left\langle\nabla_{\frac{\partial}{\partial s}} v \mid \tau_{\Phi}(f)\right\rangle-\left\langle v \mid \nabla_{\frac{\partial}{\partial s}} \nabla_{e_{i}}\left(\bar{\partial}_{i, j} \Phi\left(d f_{s}^{*} d f_{s}\right) d F e_{j}\right)\right\rangle \\
= & -\left\langle\nabla_{\frac{\partial}{\partial s}} v \mid \tau_{\Phi}(f)\right\rangle-\left\langle v \mid R_{w, d f e_{i}}\left(\bar{\partial}_{i, j} \Phi\left(d f^{*} d f\right) d f e_{j}\right)\right\rangle \\
& -\left\langle v \mid \nabla_{e_{i}} \nabla_{\frac{\partial}{\partial s}}\left(\bar{\partial}_{i, j} \Phi\left(d f_{s}^{*} d f_{s}\right) d F e_{j}\right)\right\rangle
\end{aligned}
$$

where $R$ denotes the curvature tensor of $V$. The last term is 


$$
\begin{aligned}
& -\left\langle v \mid \nabla_{e_{i}} \nabla_{\frac{\partial}{\partial s}}\left(\bar{\partial}_{i, j} \Phi\left(d f_{s}^{*} d f_{s}\right) d F e_{j}\right)\right\rangle \\
= & -\left\langle v \mid \nabla_{e_{i}}\left(\frac{d \bar{\partial}_{i, j} \Phi\left(d f_{s}^{*} d f_{s}\right)}{d s} d f e_{j}+\bar{\partial}_{i, j} \Phi\left(d f^{*} d f\right) \nabla_{\frac{\partial}{\partial s}} d F e_{j}\right)\right\rangle \\
= & -\left\langle v \mid \nabla_{e_{i}}\left(\bar{\partial}_{k, l} \bar{\partial}_{i, j} \Phi\left(d f^{*} d f\right)\left\langle\nabla_{\frac{\partial}{\partial s}} d F e_{k} \mid d f e_{l}\right\rangle d f e_{j}+\left(\bar{\partial}_{i, j} \Phi\left(d f^{*} d f\right) \nabla_{e_{j}} w\right)\right)\right\rangle \\
= & -\left\langle v \mid \nabla_{e_{i}}\left(\bar{\partial}_{k, l} \bar{\partial}_{i, j} \Phi\left(d f^{*} d f\right)\left\langle\nabla_{e_{k}} w \mid d f e_{l}\right\rangle d f e_{j}+\left(\bar{\partial}_{i, j} \Phi\left(d f^{*} d f\right) \nabla_{e_{j}} w\right)\right)\right\rangle \\
= & +\bar{\partial}_{k, l} \bar{\partial}_{i, j} \Phi\left(d f^{*} d f\right)\left\langle\nabla_{e_{i}} v \mid d f e_{j}\right\rangle\left\langle\nabla_{e_{k}} w \mid d f e_{l}\right\rangle+\bar{\partial}_{i, j} \Phi\left(d f^{*} d f\right)\left\langle\nabla_{e_{i}} v \mid \nabla_{e_{j}} w\right\rangle
\end{aligned}
$$

where the last identity holds only up to divergence.

Proposition 2.3. The second variation of the $\Phi$-energy at a $\Phi$-harmonic map $f$ is the integral over

$$
\begin{aligned}
I_{\Phi}(f)(v, w)= & -\left\langle v \mid R_{w, d f e_{i}}\left(\bar{\partial}_{i, j} \Phi\left(d f^{*} d f\right) d f e_{j}\right)\right\rangle \\
& +\bar{\partial}_{k, l} \bar{\partial}_{i, j} \Phi\left(d f^{*} d f\right)\left\langle\nabla_{e_{i}} v \mid d f e_{j}\right\rangle\left\langle\nabla_{e_{k}} w \mid d f e_{l}\right\rangle \\
& +\bar{\partial}_{i, j} \Phi\left(d f^{*} d f\right)\left\langle\nabla_{e_{i}} v \mid \nabla_{e_{j}} w\right\rangle
\end{aligned}
$$

for any vector fields $v, w$ along $f$.

We finally compute the leading symbol of the second variation. We have

$$
\frac{d^{2}}{d s d t} E_{\Phi}\left(f_{s, t}\right)=\int_{M}\langle v \mid P w\rangle d \mathrm{vol}_{g}
$$

with a symmetric second order partial differential operator $P$ acting on vector fields along $f$, i.e. on sections $v, w$ of $f^{*} T V \rightarrow M$. The restriction $P^{\perp f}$ of $P$ (or of the bilinear form given by (2.4)) to the orthogonal complement of the image of $d f: T M \rightarrow$ $f^{*} T V$ will be called second variation perpendicular to $f$. The leading symbol of $P$ is determined by the highest order term

$$
-\left\langle v \mid \bar{\partial}_{k, l} \bar{\partial}_{i, j} \Phi\left(d f^{*} d f\right)\left\langle\nabla_{e_{i}} \nabla_{e_{k}} w \mid d f e_{l}\right\rangle d f e_{j}+\bar{\partial}_{i, j} \Phi\left(d f^{*} d f\right) \nabla_{e_{i}} \nabla_{e_{j}} w\right\rangle
$$

in Proposition 2.3. Hence we get

Proposition 2.5. The leading symbol of the second variation of the $\Phi$-energy is

$$
\sigma(\xi)=\bar{\partial}_{k, l} \bar{\partial}_{i, j} \Phi\left(d f^{*} d f\right) \xi_{i} \xi_{k} d f e_{l} \otimes d f e_{j}+\bar{\partial}_{i, j} \Phi\left(d f^{*} d f\right) \xi_{i} \xi_{J}
$$

for $\xi=\sum_{i} \xi_{i} e_{i}$. Thus

$$
\langle\sigma(\xi) w \mid w\rangle=\bar{\partial}_{k, l} \bar{\partial}_{i, j} \Phi\left(d f^{*} d f\right) \xi_{i} \xi_{k}\left\langle w \mid d f e_{l}\right\rangle\left\langle w \mid d f e_{j}\right\rangle+\bar{\partial}_{i, j} \Phi\left(d f^{*} d f\right) \xi_{i} \xi_{j}\|w\|^{2}
$$

for $\xi \in T_{p} M^{*}$ and $w \in\left(f^{*} T V\right)_{p}$.

REMARK. Let $\phi: M(n \times k) \rightarrow \mathbb{R}_{0}^{+}$be a polynomial function, invariant under the action of $O(n) \times O(k)$, i.e. such that $\phi(B X A)=\phi(X)$ for all $B \in O(k), A \in O(n)$ and $X \in M(n \times k)$. For any $X \in M(n \times k)$ we can diagonalize $X^{*} X$ and find othogonal 
matrices $B$ and $A$ as before such that

$$
B X A=\left(\begin{array}{ccc}
\lambda_{1} & & 0 \\
& \ddots & \\
0 & & \lambda_{q} \\
0 & \cdots & 0
\end{array}\right) \quad \text { or } \quad\left(\begin{array}{cccc}
\lambda_{1} & & 0 & 0 \\
& \ddots & & \vdots \\
0 & & \lambda_{q} & 0
\end{array}\right)
$$

as $q:=\min \{n, k\}=n$ or $q=k$. Hence $\phi(X)=\phi\left(\lambda_{1}, \ldots, \lambda_{q}\right)$ is a symmetric polynomial and since $\phi\left( \pm \lambda_{1}, \ldots, \pm \lambda_{q}\right)=\phi\left(\lambda_{1}, \ldots, \lambda_{q}\right)$ this does not involve odd powers of the $\lambda_{i}$. Thus we find a symmetric polynomial $\Phi$ in $n$ variables such that $\phi\left(\lambda_{1}, \ldots, \lambda_{q}\right)=$ $\Phi\left(\lambda_{1}^{2}, \ldots, \lambda_{q}^{2}, 0, \ldots, 0\right)$. This extends to a polynomial $\Phi: M(n \times n)^{+} \rightarrow \mathbb{R}_{0}^{+}$such that $\phi(X)=\Phi\left(X^{*} X\right)$. For analytic $\phi$ this construction yields an analytic function $\Phi$.

Note that if $\phi$ is differentiable we do not necessarily get a differentiable function $\Phi$ with the above properties. In general $\Phi$ is only differentiable on the set of matrices of full rank $q$. For instance $\phi(X):=\operatorname{det}\left(X^{*} X\right)^{3 / 4}$ is differentiable but $\Phi(A):=\operatorname{det}(A)^{3 / 4}$ is not.

For polynomial $\phi$ there are polynomials $\Phi^{s}$ and $\Phi^{\sigma}$ such that

$$
\phi(X)=\Phi\left(X^{*} X\right)=\Phi^{s}\left(s_{1}, \ldots, s_{q}\right)=\Phi^{\sigma}\left(\sigma_{1}, \ldots, \sigma_{q}\right)
$$

where $\sigma_{l}$ is the $l$ th elementary symmetric polynomial in the eigenvalues $\lambda_{1}^{2}, \ldots, \lambda_{q}^{2}$ of $X^{*} X$ determined by

$$
\sum_{l=0}^{n} \sigma_{l}\left(X^{*} X\right) t^{l}=\operatorname{det}\left(1+t X^{*} X\right)
$$

and

$$
s_{k}=\sum_{l=0}^{n} \lambda_{l}^{2 q}=\operatorname{Tr}\left(\left(X^{*} X\right)^{l}\right) .
$$

In the smooth case one can use a theorem of Glaeser, [8], to get smooth functions $\Phi^{s}$ and $\Phi^{\sigma}$.

\section{Applications.}

3.1. Isometric immersions. For isometric immersions the preceding formulae simplify substantially. By invariance $d \Phi(i d)$ must be some multiple $\lambda \operatorname{Tr}$ of the trace. We have the following.

THEOREM 1. Let $f: M \rightarrow V$ be an isometric immersion and assume that $d \Phi($ id $) \neq 0$. Then

1. $f$ is $\Phi$-harmonic if and only if it is harmonic.

2. If $\lambda>0$ then the leading symbol of $P^{\perp f}$ is positive definite, hence the second variation perpendicular to $f$ has finite index.

Proof. (1) For an isometric immersion or a Riemannian submersion the first term in (2.2) vanishes. Since an isometric immersion $f$ has $d f^{*} d f=i d$ we get

$$
\tau_{\Phi}(f)=\bar{\partial}_{i, j} \Phi(i d) \nabla_{e_{i}} d f e_{j}=2 \lambda \operatorname{Tr} \nabla d f=2 \lambda \tau(f) .
$$


(2) On vector fields $w$ normal to $f$, i.e perpendicular to the $d f e_{l}$ in (2.6), the first summand in (2.6) vanishes. As before the second summand is some multiple of the trace which shows that

$$
\sigma(\xi)=\bar{\partial}_{i, j} \Phi\left(d f^{*} d f\right) \xi_{i} \xi_{j}=2 \lambda\|\xi\|^{2}>0
$$

for $\xi \neq 0$. Thus the restriction of $P$ to (Image $(d f))^{\perp} \subset f^{*} T V$ is elliptic with positive definite leading symbol and therefore has only finitely many negative eigenvalues.

3.2. Stability of isometries. By invariance, the second derivative $d^{2} \Phi(i d)$ is a homogeneous polynomial of degree 2 . Therefore there are $\mu, v \in \mathbb{R}$ such that

$$
d^{2} \Phi(i d)(H)=\mu \operatorname{Tr}\left(H^{2}\right)+v(\operatorname{Tr} H)^{2}
$$

The second variation formula in Proposition 2.3 simplifies to

$$
\begin{aligned}
I_{\Phi}(f)(v, v)= & -\left\langle v \mid R_{v, e_{i}}\left(\bar{\partial}_{i, j} \Phi(i d) e_{j}\right)\right\rangle \\
& +\bar{\partial}_{k, l} \bar{\partial}_{i, j} \Phi(i d)\left\langle\nabla_{e_{i}} v \mid e_{j}\right\rangle\left\langle\nabla_{e_{k}} v \mid e_{l}\right\rangle+\bar{\partial}_{i, j} \Phi(i d)\left\langle\nabla_{e_{i}} v \mid \nabla_{e_{j}} v\right\rangle \\
= & -2 \lambda \operatorname{Ric}(v) \\
& +\mu\left(\left\langle\nabla_{e_{i}} v \mid e_{j}\right\rangle+\left\langle\nabla_{e_{j}} v \mid e_{i}\right\rangle\right)^{2} \\
& +4 v\left\langle\nabla_{e_{i}} v \mid e_{i}\right\rangle\left\langle\nabla_{e_{k}} v \mid e_{k}\right\rangle+2 \lambda\left\langle\nabla_{e_{i}} v \mid \nabla_{e_{i}} v\right\rangle \\
= & -2 \lambda \operatorname{Ric}(v) \\
& +2 \mu\left(\|\nabla v\|^{2}+\operatorname{Tr}\left((\nabla v)^{2}\right)\right)+4 v(\operatorname{div}(v))^{2}+2 \lambda\|\nabla v\|^{2} \\
= & -2 \lambda \operatorname{Ric}(v)+2(\mu+\lambda)\|\nabla v\|^{2}+2 \mu \operatorname{Tr}\left((\nabla v)^{2}\right)+4 v(\operatorname{div}(v))^{2} \\
= & -2 \lambda \operatorname{Ric}(v)+\mu\left\|L_{v} g\right\|^{2}+4 v(\operatorname{div}(v))^{2}+2 \lambda\|\nabla v\|^{2}
\end{aligned}
$$

since $\operatorname{Tr}(\nabla v)=\operatorname{div}(v)$. Comparing this with the Bochner formula (see e.g. [10]):

$$
\int_{M}-\operatorname{Ric}(v)-\frac{1}{2}\left\|L_{v} g\right\|^{2}+(\operatorname{div}(v))^{2}+\|\nabla v\|^{2}=0
$$

we obtain

TheOrem 2. Assume that $\mu \geq-\lambda$ and that $2 v \geq \lambda$. Then any isometry of $M$ is Ф-stable.

We now derive a sufficient criterion for the identity map on a sphere to be unstable. To that end let $v$ be the gradient vectorfield on $S^{n} \subset \mathbb{R}^{n+1}$ of the restriction of a linear $\operatorname{map} p: \mathbb{R}^{n+1} \rightarrow \mathbb{R}, p(x)=\langle p, x\rangle$ for a unit vector $p \in \mathbb{R}^{n+1}$ as in [9]. Then $\|v(x)\|^{2}+$ $p(x)^{2}=1$ and $\nabla_{x} v=-p x$ for all $x \in T S^{n}$, hence $\left\langle\nabla_{e_{i}} v, e_{j}\right\rangle=-p \delta_{i, j}$. Since the Ricci curvature of $S^{n}$ is $\operatorname{Ric}(v)=(n-1)\|v\|^{2}$, the formula for the index form yields

$$
I_{\Phi}(v, v)=-2 \lambda(n-1)\|v\|^{2}+\left(4 \mu n+4 v n^{2}+2 \lambda n\right) p^{2} .
$$

Denoting by $\omega_{n-1}$ the volume of the standard $(n-1)$-sphere we compute 


$$
\begin{aligned}
\int_{S^{n}}\|v\|^{2} & =\omega_{n-1} \int_{-\pi / 2}^{\pi / 2} \cos (\theta)^{n+1} d \theta \\
& =\omega_{n-1}\left(\left[\sin (\theta) \cos (\theta)^{n}\right]_{-\pi / 2}^{\pi / 2}+\int_{-\pi / 2}^{\pi / 2} \sin (\theta)^{2} \cos (\theta)^{n-1} d \theta\right) \\
& =n \int_{S^{n}} p^{2} .
\end{aligned}
$$

Inserting this into (3.2) shows the following

THEOREM 3. If

$$
\lambda(n-2)>2 \mu+2 v n
$$

then id: $S^{n} \rightarrow S^{n}$ is $\Phi$-unstable.

3.3. Examples. For some of the functionals mentioned in the introduction theorems 2 and 3 give:

1. For the $p$-energy, $\Phi(A)=(\operatorname{Tr}(A))^{p}$ we compute $\lambda=p n^{p-1}, \mu=0$ and $v=$ $p(p-1) n^{p-2}$. Thus $i d_{S^{n}}$ is unstable if $n>2 p$. Isometries are generally stable if $n \leq 2(p-1)$.

2. The exponential energy, $\Phi(A)=e^{\operatorname{Tr} A}$, has $\lambda=e^{n}, \mu=0, v=e^{n}$. Thus isometries are always stable for $E_{\Phi}$. This is the proof of [2].

3. For $\Phi(A)=\operatorname{Tr}\left(A^{p}\right)$ we get $\lambda=p, \mu=p(p-1)$ and $v=0$. Thus $i d_{S^{n}}$ is unstable if $n>2 p$.

4. For $\Phi(A)=\operatorname{Tr} \exp (A)$ we get $\lambda=e, \mu=e, v=0$. Therefore $i d_{S^{n}}$ is unstable if $n>4$.

5. For $\Phi(A)=\operatorname{det}(A)$ we get $\lambda=1, \mu=-1, v=1$. Thus any isometry is stable for $E_{\mathrm{det}}$.

6. Let $\alpha_{1}, \ldots, \alpha_{n}$ be the eigenvalues of $A$ and if $n \geq 2$ define the discriminant $\Phi(A):=\prod_{1 \leq i<j \leq n}\left(\alpha_{i}-\alpha_{j}\right)^{2}$. Then $E_{\Phi}$ has $\lambda=\mu=v=0$ and the second variation at an isometry vanishes.

\section{REFERENCES}

1. M. Ara, Geometry of F-harmonic maps, Kodai Math. J. 22 (1999), No. 2, 243-263.

2. L.-F. Cheung and P.-F. Leung, The second variation formula for exponentially harmonic maps, Bull. Austral. Math. Soc. 59 (1999), No. 3, 509-514.

3. L.-F. Cheung and P. F. Leung, Some results on stable $p$-harmonic maps, Glasgow Math. J. 36 (1994), No. 1, 77-80.

4. J. Eells and L. Lemaire, Selected topics in harmonic maps, CBMS Regional Conference Series in Mathematics No. 50.

5. J. Eells and L. Lemaire, A report on harmonic maps, Bull. London Math. Soc. 10 (1978), No. 1, 1-68.

6. J. Eells and L. Lemaire, Another report on harmonic maps, Bull. London Math. Soc. 20 (1988), No. 5, 385-524.

7. J. Eells and L. Lemaire, Some properties of exponentially harmonic maps in Partial differential equations, Part 1, 2 (Warsaw, 1990), (Banach Center Publ., 27, Part 1, 2, Polish Acad. Sci., Warsaw, 1992), 129-136.

8. G. Glaeser, Fonctions composées differentiables, Ann. of Math. (2) 77 (1963), 193-209.

9. Y. Xin, Geometry of harmonic maps, Progress in Nonlinear Differential Equations and their Applications, 23 (Birkhäuser Boston, Inc., Boston, MA, 1996).

10. K. Yano, Integral formulas in Riemannian geometry, Pure and Applied Mathematics, No. 1 (Marcel Dekker, Inc., New York 1970). 